\title{
Exploiting GPUs to investigate an inversion method that retrieves cardiac conductivities from potential measurements
}

\author{
Barbara M. Johnston ${ }^{1} \quad$ Josef P. Barnes ${ }^{2}$
}

(Received 16 December 2013; revised 7 March 2014)

\begin{abstract}
Accurate cardiac bidomain conductivity values are essential for realistic simulation of various cardiac electrophysiological phenomena. A method was previously developed that can determine the conductivities from measurements of potential on a multi-electrode array placed on the surface of the heart. These conductivities, as well as a value for fibre rotation, are determined using a mathematical model and a two-pass process that is based on Tikhonov regularisation. Using simulated potentials, to which noise is added, the inversion method was recently shown to retrieve the intracellular conductivities accurately with up to $15 \%$ noise and the extracellular conductivities extremely accurately even with $20 \%$ noise. Recent work investigated the sensitivity of the
\end{abstract}

http://journal.austms.org.au/ojs/index.php/ANZIAMJ/article/view/7790 gives this article, (c) Austral. Mathematical Soc. 2014. Published March 21, 2014, as part of the Proceedings of the 11th Biennial Engineering Mathematics and Applications Conference. ISSN 1446-8735. (Print two pages per sheet of paper.) Copies of this article must not be made otherwise available on the internet; instead link directly to this URL for this article. 
method to the choice of the regularisation parameters. Such a study only became possible due to modifications that were made to the $\mathrm{C}++$ code so that it could run on graphical processing units (GPUs) on the CUDA platform. As the method required the solution of a large number of matrix equations, the highly parallel nature of GPUs was exploited to accelerate execution of the code. Reorganisation of the code and more efficient memory management techniques allowed the data to completely fit in the GPU memory. Comparison between the execution time on the GPU versus the original CPU code shows a speedup of up to 60 times. In the future, the speedup could be further increased with greater use of shared memory, which has a much lower latency (access time) than global memory.

\section{Contents}

1 Introduction

2 Simulations and inversion method

2.1 Model and solution technique . . . . . . . . . C20

2.2 Array, inversion method and simulations . . . . . . . C22

3 Parallelisation

3.1 CPU parallelisation . . . . . . . . . . . . C23

3.2 GPU parallelisation . . . . . . . . . . . . . . . C24

3.3 Performance improvement . . . . . . . . . . . C25

4 Results and discussion

5 Conclusions

References 


\section{Introduction}

Simulation studies, using mathematical modelling, are increasingly being used to explore the mechanisms behind heart disorders such as myocardial ischaemia and atrial and ventricular fibrillation [1]. However, realistic simulations require accurate model parameters. The bidomain model [2], which is commonly used to simulate the electric field in cardiac tissue, uses six cardiac conductivity values, in addition to other parameters. These conductivities are necessary because electrical current flows in three directions in cardiac tissue: along (longitudinal) and across (transverse) the fibres of cardiac cells that make up the sheets of cardiac tissue, as well as between (normal) the sheets. This occurs in the two interpenetrating domains (intracellular and extracellular) of the bidomain model.

There are a number of theoretical and practical difficulties associated with making and interpreting the measurements necessary to find the cardiac conductivities. The result of this is that only three sets $[3,4,5]$ of four conductivity values were experimentally determined (where the assumption made is that the conductivities in the transverse and normal directions are equal), while the two sets of six conductivities that are available [6, 7] are not fully experimentally determined. It was found that the four conductivity datasets are not consistent [8] and produce results that are open to considerable interpretation [9], as is also the case for the six conductivity sets [10]. In addition, experimental work $[6,11]$ showed that ventricular tissue does have three distinct propagation directions, which led to renewed efforts by researchers to develop techniques for finding cardiac conductivity values $[12,13,14,15]$.

These efforts include the presentation of a model and solution method as well as a multi-electrode measuring array $[16,17,18]$ which were used in simulations with synthetic data to demonstrate that it is possible to accurately retrieve the six bidomain conductivities. The validation consisted of adding noise of up to $5 \%$ to simulated measurements of potential on the array and then using 
an inversion technique to retrieve the conductivities. The inversion technique uses a two-pass process based on the so-called four electrode technique [19], where measurements are made on the outer pair of four equi-spaced collinear electrodes, after current is applied to the other two. The first pass uses 'closely-spaced' electrodes to retrieve the extracellular conductivities and the second pass uses more 'widely-spaced' electrodes to retrieve the intracellular conductivities (and the fibre rotation angle, which is associated with the rotation of the sheets of cardiac fibres). The inversion process is iterative and fits the conductivities by minimising a Tikhonov functional [17, 18].

Since the solution method for the model is amenable to parallelisation, it recently became possible to consider whether the conductivities can still be retrieved accurately when much larger values of noise are added to the potential measurements. In addition, other aspects of the inversion process can now be studied to see if it is possible to retrieve the conductivities, particularly the intracellular ones, more accurately. These two considerations are the focus of this article and the results of these investigations are presented in Section 4, along with an outline of the previously presented $[17,18]$ mathematical model, its solution method, the measuring array and the inversion method in Section 2 and a discussion of the CPU and GPU parallelisation in Section 3.

\section{Simulations and inversion method}

\subsection{Model and solution technique}

Cardiac tissue consists of sheets of fibres that rotate, relative to one another, through the tissue. Also, current is able to flow more easily along the fibres ( $l=$ longitudinal) than across the fibres within the sheet $(\boldsymbol{t}=$ transverse) or between the sheets $(\mathrm{n}=$ normal $)$. This leads to six cardiac bidomain ( $i=$ intracellular, $e=$ extracellular domain) conductivity values $g_{p q}$ where $p=$ $i, e$ and $\mathrm{q}=\mathrm{l}, \mathrm{t}, \mathrm{n}$. 
Here, the electric potential in a block of cardiac ventricular tissue and its adjacent blood mass is modelled using the bidomain equations [2], together with Laplace's equation in the blood (b):

$$
\nabla \cdot \mathbf{M}_{\mathrm{i}} \nabla \phi_{\mathrm{i}}=\frac{\beta}{\mathrm{R}}\left(\phi_{\mathrm{i}}-\phi_{e}\right), \quad \nabla \cdot \mathbf{M}_{e} \nabla \phi_{e}=-\frac{\beta}{\mathrm{R}}\left(\phi_{\mathrm{i}}-\phi_{e}\right)-\mathrm{I}_{\mathrm{s}}, \quad \nabla^{2} \phi_{\mathrm{b}}=0,
$$

where $\phi_{p}$ with $p=i, e, b$ is the potential, $\beta$ is the surface to volume ratio of the cells, $R$ is the membrane resistance and $I_{s}$ is the applied current. The conductivity tensors $\mathbf{M}_{\mathfrak{p}}$, with $p=i, e$, take account of the anisotropic conductivity, as well as the fibre rotation, which is assumed to be linear through an angle of $\alpha$ between the epicardium and the endocardium. Johnston et al. $[17,20]$ provided a full description of the model and the boundary conditions used, as well as the solution technique.

The model is solved by first expanding $\phi_{p}$ with $p=i, e$ as a Fourier series, where

$$
\begin{aligned}
\phi_{\mathrm{p}}(x, y, z)= & \sum_{\mathrm{r}=0}^{\infty} \sum_{s=0}^{\infty}\left[C_{r s}^{\mathrm{p}}(z) \cos (s \pi y) \cos (\mathrm{r} \pi x)+\mathrm{D}_{\mathrm{rs}}^{\mathrm{p}}(z) \sin (\mathrm{s} \pi \mathrm{y}) \cos (\mathrm{r} \pi x)\right. \\
& \left.+\mathrm{E}_{\mathrm{rs}}^{\mathrm{p}}(z) \cos (\mathrm{s} \pi \mathrm{y}) \sin (\mathrm{r} \pi x)+\mathrm{F}_{\mathrm{rs}}^{\mathrm{p}}(z) \sin (\mathrm{s} \pi \mathrm{y}) \sin (\mathrm{r} \pi x)\right]
\end{aligned}
$$

This results in two sets of four ordinary differential equations in the coefficients $X_{r s}^{p}(z)$, with $X=C, D, E, F$. The coefficients are found by solving a banded system of linear algebraic equations, which are the result of a one dimensional finite difference scheme in the $z$ direction.

The parameters used to solve the model are [21] $\mathrm{g}_{\mathrm{b}}=6.7 \mathrm{mS} / \mathrm{cm}, \beta=$ $2000 \mathrm{~cm}^{-1}, \mathrm{R}=9100 \Omega \mathrm{cm}^{2}, \mathrm{I}_{\mathrm{s}}=50 \mu \mathrm{A} / \mathrm{mm}^{3}$ and $\alpha=2 \pi / 3$, as well as two sets of six conductivity values, those of Hooks et al. [6] and MacLachlan et al. [7] (Table 1). 
Table 1: Conductivity data (in $\mathrm{mS} / \mathrm{cm}$ ) from the indicated studies.

\begin{tabular}{l|llllll} 
Study & $g_{e l}$ & $g_{\text {et }}$ & $g_{\text {en }}$ & $g_{i l}$ & $g_{i t}$ & $g_{\text {in }}$ \\
\hline Hooks et al. [6] & 2.63 & 2.45 & 1.087 & 2.63 & 0.263 & 0.08 \\
MacLachlan et al. [7] & 2.0 & 1.65 & 1.3514 & 3.0 & 1.0 & 0.35125
\end{tabular}

\subsection{Array, inversion method and simulations}

The approach used to find the required parameters (six conductivities and fibre rotation) is to make potential measurements on the heart surface using an electrode array and then to use an inversion technique to recover the original parameters. Since the potentials depend non-linearly on the parameters (first two equations in (1)), and there will be measurement noise, the inversion technique involves minimising a Tikhonov functional, using the SolvOpt solver [22]. For the first pass, which retrieves the extracellular conductivities, this is given by

$$
f_{1}=\sum_{i=0}^{24}\left[\phi_{M}(i)-\phi_{C}(i)\right]^{2}+\gamma_{1}^{2}\left[g_{i l}^{2}+g_{i t}^{2}+g_{i n}^{2}+g_{e l}^{2}+g_{e t}^{2}+g_{e n}^{2}\right]+\gamma_{2}^{2} \alpha^{2}
$$

where $\gamma_{1}=10^{-2}$ and $\gamma_{2}=10^{-5}$ are the regularisation parameters and the initial value used for $\alpha=1$, while the conductivities are taken to be $1 \times 10^{-3} \mathrm{~S} / \mathrm{cm}$, except for $g_{i n}=1 \times 10^{-4} \mathrm{~S} / \mathrm{cm}$. Here $\phi_{M}$ is the difference in measured potential between electrode $i$ and the reference electrode and $\phi_{C}$ is similar but for the calculated value at each iteration of the solver. These potentials are measured using the inner (green) electrodes of the multielectrode array shown in Figure 1. A similar Tikhonov functional is used in the second pass [17], except that the extracellular values found in the first pass are held constant, and only the intracellular conductivities and fibre rotation are solved for (in this case using all the electrodes, except for the two used for current injection and retrieval).

Sets of 15 simulations are produced for each pass, noise level and conductivity set. This is achieved by using the model to calculate the potentials at the 


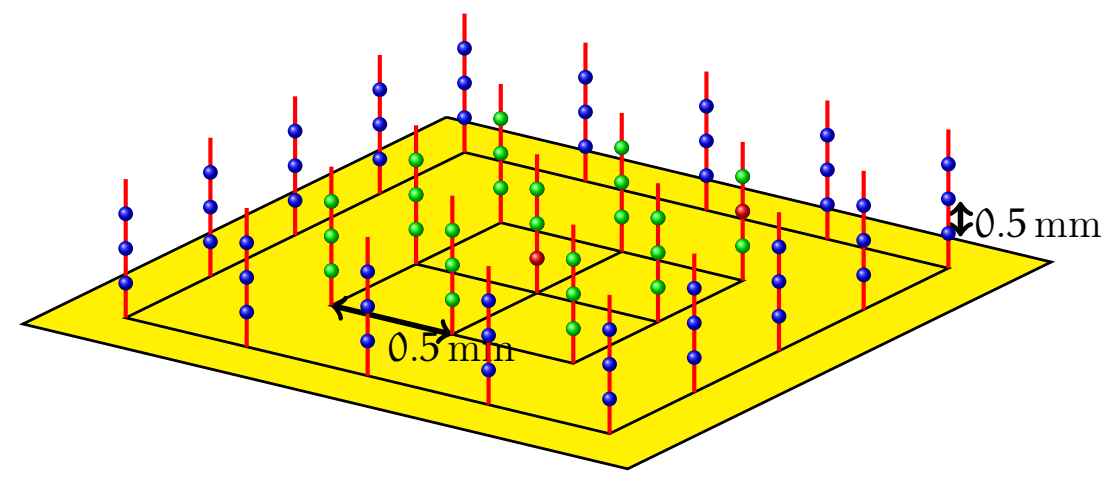

Figure 1: The electrode array used to make the potential measurements. A subset of the electrodes (green) is used in the first pass and all electrodes are used in the second pass. The red electrodes are current source and sink electrodes.

appropriate set of measuring electrodes on the array, adding noise to these values and then using the inversion technique to retrieve the conductivities and fibre rotation in a two-pass process [17]. However, since each first pass inversion requires about 80-100 iterations of the solver and each second pass inversion requires another $30-40$ iterations, this process is extremely time consuming. Fortunately, the structure of the problem is such that it is amenable to parallelisation.

\section{Parallelisation}

\subsection{CPU parallelisation}

Solving the model involves the sum of a set of calculations that involve $X_{\mathrm{rs}}^{\mathrm{p}}$ with $p=i, e$ and $X=C, D, E, F$ (see equation (2)). Since these are independent for each $r, s$ combination, it is straightforward to parallelise the $\mathrm{C}++$ code to 
run on multiple CPUs. For example, for four CPUs, with $r, s=0,1, \ldots, 200$, the loops are split with $0 \leqslant r \leqslant 200$ on each processor and then $0 \leqslant s \leqslant 49$ on CPU $0, \ldots, 150 \leqslant s \leqslant 200$ on CPU3. The results from each of the four processors is then sent to the root CPU (say CPU0) to be added together to give the final result. The freely available MPI (message passing interface) library MPICH2 is used to perform the dividing and communicating tasks.

\subsection{GPU parallelisation}

The next step is to modify the code so that it can be run in parallel on the CUDA platform, but this presents challenges due to the memory restrictions that are associated with GPUs. CPUs have a small number of fast cores called ALUs (arithmetic logic units), on which each parallel section is run. GPUs, on the other hand, have hundreds of parallel threads (the code that is run on a single ALU) arranged in blocks, but only a limited amount of shared memory per block. This is overcome by using global memory (DRAM), but this has a much higher latency (access time) than shared memory. The challenge is to balance these two aspects of memory access.

An initial problem is that any one of the main matrices or vectors used in the code is too large to be stored in shared memory. In addition, the amount of global memory required per thread (around $180 \mathrm{kB}$ for 200 discretisation points in the finite difference scheme) is also too large. These problems are overcome by some creative reorganisation of the code that takes advantage of the fixed bandwidth of the matrices. The new code uses temporary storage of vectors and Gaussian elimination, rather than a banded solution technique, to achieve a $65 \%$ reduction in memory per thread, making it possible for all the data to fit on the GPU memory.

When using GPUs, allocating the data to the processors is easier than for CPUs since each thread is a single $r, s$ combination. These are then copied back to the $\mathrm{CPU}$, which is responsible for adding them together and displaying the final result for the potential. 


\subsection{Performance improvement}

The CPU hardware used in this work is an Intel S2011 Core i7 3930K with a clock speed of $3.20 \mathrm{GHz}$ and the GPU is the Nvidia GTX690 (4 GB, 3072 cores, dual GPU). The original kernel (for a forward pass with 200 points in each direction), which runs on a single CPU in approximately $29 \mathrm{~s}$, ran on a single GPU in approximately $0.5 \mathrm{~s}$, giving a speedup of around 60 times. In practice, the system actually consisted of two of these GPUs plus another GTX660 card, which could be utilised for these calculations if it was not being used for visualisation, giving a run-time of around $0.23 \mathrm{~s}$. In terms of the simulations that retrieved the conductivities, this translated from a single first pass (of around 80-100 iterations that took around 12-14 hours) to a full set of 15 first pass simulations (that took less than two hours).

\section{Results and discussion}

Percentage relative errors \pm one standard deviation, averaged over 15 runs, for noise levels 5\%, 10\%, 15\% and 20\%, are given in Table 2 for the datasets of Hooks et al. [6] and MacLachlan et al. [7]. These errors show that, even with up to $20 \%$ added noise, the three extracellular conductivities $g_{e l}, g_{e t}$ and $g_{e n}$ are retrieved extremely accurately (at worst at around half the added error) and, with the exception of one case, with relative errors that are less than $3 \%$. The intracellular conductivities and fibre rotation cannot be retrieved as accurately, but, at around 2-3 times the added noise, they are still found quite accurately for noise up to $15 \%$. It would seem that $20 \%$ added noise is near the limit of the algorithm since there is a problem with retrieving $g_{i t}$ for the Hooks' conductivities [6], but not the rest of the parameters or those of MacLachlan et al. [7]. It is also interesting to note that, when retrieving the second pass parameters $\left(g_{i q}\right.$ and $\alpha$ ), in many cases, it seems that three parameters are found very accurately and one is considerably less accurate, or alternatively, four parameters are found moderately accurately. It is possible 
Table 2: Percentage relative errors \pm one standard deviation, for various noise levels, when retrieving the indicated cardiac conductivities and fibre rotation using data sets: (a) Hooks et al. [6]; and (b) MacLachlan et al. [7]

Noise

\begin{tabular}{r|llll} 
& \multicolumn{4}{|c}{ Noise } \\
(a) & $5 \%$ & $10 \%$ & $15 \%$ & $20 \%$ \\
\hline$g_{\text {el }}$ & $0.5 \pm 1.9$ & $0.6 \pm 1.9$ & $0.1 \pm 3.5$ & $0.7 \pm 5.3$ \\
$g_{\text {et }}$ & $1.1 \pm 2.7$ & $0.7 \pm 3.0$ & $0.1 \pm 2.5$ & $7.2 \pm 9.2$ \\
$g_{\text {en }}$ & $0.4 \pm 2.2$ & $1.3 \pm 1.6$ & $0.6 \pm 2.1$ & $3.0 \pm 4.0$ \\
$g_{\text {il }}$ & $2.6 \pm 3.8$ & $17.7 \pm 8.2$ & $1.9 \pm 9.8$ & $2.5 \pm 10.3$ \\
$g_{\text {it }}$ & $6.5 \pm 19.4$ & $2.4 \pm 29.5$ & $1.6 \pm 30.2$ & $112 \pm 98$ \\
$g_{\text {in }}$ & $10.6 \pm 12.2$ & $3.0 \pm 16.4$ & $18.8 \pm 30.4$ & $3.6 \pm 52.5$ \\
$\alpha$ & $0.1 \pm 5.7$ & $10.5 \pm 17.0$ & $15.9 \pm 28.8$ & $1.4 \pm 29.9$ \\
$(b)$ & $5 \%$ & \multicolumn{3}{|c}{ Noise } \\
\hline$g_{e l}$ & $0.7 \pm 2.1$ & $10 \% \pm 6.7$ & $0.3 \pm 2.3$ & $0.6 \pm 4.2$ \\
$g_{\text {et }}$ & $2.7 \pm 3.1$ & $0.2 \pm 6.0$ & $0.2 \pm 4.7$ & $1.0 \pm 4.8$ \\
$g_{\text {en }}$ & $0.4 \pm 1.7$ & $0.2 \pm 5.2$ & $1.6 \pm 3.4$ & $0.0 \pm 2.9$ \\
$g_{\text {il }}$ & $2.1 \pm 3.0$ & $3.0 \pm 6.6$ & $8.8 \pm 9.6$ & $25.6 \pm 14.5$ \\
$g_{\text {it }}$ & $15.7 \pm 8.0$ & $9.2 \pm 18.6$ & $10.8 \pm 12.0$ & $13.7 \pm 28.2$ \\
$g_{\text {in }}$ & $1.7 \pm 7.3$ & $17.6 \pm 14.0$ & $11.8 \pm 19.3$ & $22.9 \pm 37.9$ \\
$\alpha$ & $8.2 \pm 7.9$ & $1.4 \pm 20.3$ & $8.6 \pm 18.2$ & $29.7 \pm 42.5$
\end{tabular}

that the minimisation algorithm must find a balance between the errors in the various parameters.

The speed-up in the running times for the simulations now provides the opportunity to investigate a number of aspects of the inversion algorithm in a systematic fashion. Here the same sets of noisy potentials are used each time and only some aspect of the inversion is changed. For example, the sensitivity of the retrievals to the size of the regularisation parameters is examined and it is confirmed that the algorithm is quite insensitive to the size of $\gamma_{1}$ and $\gamma_{2}$ in the Tikhonov regularisation (for example, equation (3)). It is also confirmed 
that the algorithm is not particularly dependent on initial values used in the first pass, although this is not the case for the second pass. However, even using exact values for the extracellular conductivities in the second pass does not produce second pass parameters that are markedly more accurate than those above and this seems to be related to the noise in the potentials.

\section{Conclusions}

Results from a simulation study showed that it is possible to accurately retrieve the six bidomain cardiac conductivity values and fibre rotation from measurements of potential made on an electrode array on the heart's surface, when noise of up to at least 15\% is added. Extracellular conductivities, retrieved from potentials with up to $20 \%$ noise added have relative errors that are between 0.25 and 0.5 of the added noise, while reasonably accurate intracellular conductivities and fibre rotation are found for noise up to $15 \%$. Innovative data management and memory access techniques made it possible for the $\mathrm{C}++$ code to be run on GPUs and achieve speed-ups of up to 60 times, which has opened up the possibility of studying other aspects of the retrieval algorithm, including some mentioned below. It also made it feasible to process the large amounts of data that will be produced when this method is applied in vivo. Future work on the computing aspect of the project could include further reorganisation of the code to increase the use of shared memory.

Strategies that were investigated for reducing the overall error in the intracellular conductivities and fibre rotation, which were retrieved in the second pass, included: adjusting the regularisation parameters; varying the initial values used in the first pass, and attempting to find the extracellular values that are used in the second pass more accurately. One final conclusion from these studies is that, because noise is added to the potentials, it does not seem to be possible to get the overall error in the intracellular conductivities and fibre rotation below a certain level. 


\section{References}

[1] Clayton, R. H., Bernus, O., Cherry, E. M., Dierckx, H., Fenton, F. H., Mirabella, L., Panfilov, A. V., Sachse, F. B., Seemann, G., and Zhang, H. Models of cardiac tissue electrophysiology: Progress, challenges and open questions. Progress in Biophysics and Molecular Biology, 104(1-3):22-48, 2011. doi:10.1016/j.pbiomolbio.2010.05.008 C19

[2] Arthur, R. M. and Geselowitz, D. B. Effect of inhomogeneities on the apparent location and magnitude of a cardiac current dipole source. IEEE Transactions on Biomedical Engineering, 17:141-146, 1970. doi:10.1109/TBME.1970.4502713 C19, C21

[3] Clerc, L. Directional differences of impulse spread in trabecular muscle from mammalian heart. Journal of Physiology, 255:335-346, 1976. http://jp.physoc.org/content/255/2/335 C19

[4] Roberts, D. E., Hersh, L. T., and Scher, A. M. Influence of cardiac fiber orientation on wavefront voltage, conduction velocity and tissue resistivity in the dog. Circ. Res., 44:701-712, 1979. doi:10.1161/01.RES.44.5.701 C19

[5] Roberts, D. E. and Scher, A. M. Effects of tissue anisotropy on extracellular potential fields in canine myocardium in situ. Circ. Res., 50:342-351, 1982. doi:10.1161/01.RES.50.3.342 C19

[6] Hooks, D. A. Myocardial segment-specific model generation for simulating the electrical action of the heart. BioMedical Engineering OnLine, 6(1):21-21, 2007. doi:10.1186/1475-925X-6-21 C19, C21, C22, $\mathrm{C} 25, \mathrm{C} 26$

[7] MacLachlan, M. C., Sundnes, J., and Lines, G. T. Simulation of ST segment changes during subendocardial ischemia using a realistic 3-D cardiac geometry. IEEE Transactions on Biomedical Engineering, 
52(5):799-807, 2005. doi:10.1109/TBME.2005.844270 C19, C21, C22, $\mathrm{C} 25, \mathrm{C} 26$

[8] Roth, B. J. Electrical conductivity values used with the bidomain model of cardiac tissue. IEEE Transactions on Biomedical Engineering, 44(4):326-328, April 1997. doi:10.1109/10.563303 C19

[9] Johnston, P. R. and Kilpatrick, D. The effect of conductivity values on ST segment shift in subendocardial ischaemia. IEEE Transactions on Biomedical Engineering, 50(2):150-158, February 2003. doi:10.1109/TBME.2002.807660 C19

[10] Johnston, P. R. Cardiac conductivity values - a challenge for experimentalists? Noninvasive Functional Source Imaging of the Brain and Heart $\& 2011$ 8th International Conference on Bioelectromagnetism (NFSI \& ICBEM), pages 39-43, 13-16 May 2011. doi:10.1109/NFSI.2011.5936816 C19

[11] Hooks, D. A. and Trew, M. L. Construction and validation of a plunge electrode array for three-dimensional determination of conductivity in the heart. IEEE Transactions on Biomedical Engineering, 55(2):626-635, 2008. doi:10.1109/TBME.2007.903705 C19

[12] Trew, M. L., Caldwell, B. J., Gamage, T. P. B., Sands, G. B., and Smaill, B. H. Experiment-specific models of ventricular electrical activation: Construction and application. In 30th Annual International IEEE EMBS Conference, pages 137-140, 2008. doi:10.1109/IEMBS.2008.4649109 C19

[13] Caldwell, B. J., Trew, M. L., Sands, G. B., Hooks, D. A., LeGrice, I. J., and Smaill, B. H. Three distinct directions of intramural activation reveal nonuniform side-to-side electrical coupling of ventricular myocytes. Circulation: Arrhythmia and Electropysiology, 2:433-440, 2009. doi:10.1161/CIRCEP.108.830133 C19 
[14] Pollard, A. E., Ellis, C. D., and Smith, W. M. Linear electrode arrays for stimulation and recording within cardiac tissue space constants. IEEE Transactions on Biomedical Engineering, 55(4):1408-1414, 2008. doi:10.1109/TBME.2007.912401 C19

[15] Pollard, A. E. and Barr, R. C. A biophysical model for cardiac microimpedance measurements. American Journal of Physiology-Heart and Circulatory Physiology, 298:H1699-H1709, 2010. doi:10.1152/ajpheart.01131.2009 C19

[16] Johnston, B. M. Design of a multi-electrode array to measure cardiac conductivities. ANZIAM Journal, 54:C271-C290, 2013.

http://journal . austms.org. au/ojs/index.php/ANZIAMJ/article/ viewFile/6278/1694 C19

[17] Johnston, B. M. and Johnston, P. R. A multi-electrode array and inversion technique for retrieving six conductivities from heart potential measurements. Medical and Biological Engineering and Computing, 51(12):1295-1303, 2013. doi:10.1007/s11517-013-1101-2 C19, C20, C21, C22, C23

[18] Johnston, B. M. Using a sensitivity study to facilitate the design of a multi-electrode array to measure six cardiac conductivity values Mathematical Biosciences, 244:40-46, 2013. doi:10.1016/j.mbs.2013.04.003 C19, C20

[19] Plonsey, R. and Barr, R. The four-electrode resistivity technique as applied to cardiac muscle. IEEE Transactions on Biomedical Engineering, 29(7):541-546, 1982. doi:10.1109/TBME.1982.324927 C20

[20] Johnston, B. M., Johnston, P. R., and Kilpatrick, D. A new approach to the determinination of cardiac potential distributions: Application to the analysis of electrode configurations. Mathematical Biosciences, 202(2):288-309, 2006. doi:10.1016/j.mbs.2006.04.004 C21 
[21] Johnston, B. M., Johnston, P. R., and Kilpatrick, D. Analysis of electrode configurations for measuring cardiac tissue conductivities and fibre rotation. Annals of Biomedical Engineering, 34(6):986-996, June 2006. doi:10.1007/s10439-006-9098-4 C21

[22] Kuntsevich, A. and Kappel, F. SolvOpt: The solver for Local Nonlinear Optimisation Problems, version 1.1 in C. Technical Report, Institute for Mathematics: Karl-Franzens University of Graz, 1997. http://uni-graz.at/imawww/kuntsevich/solvopt/ps/manual.pdf $\mathrm{C} 22$

\section{Author addresses}

1. Barbara M. Johnston, School of Biomolecular and Physical Science, Griffith University, Nathan, Queensland 4111, Australia. mailto:Barbara.Johnston@griffith.edu.au

2. Josef P. Barnes, School of Biomolecular and Physical Science, Griffith University, Nathan, Queensland 4111, Australia. 